\title{
Archeologie della presenza: Digital liveness, vanish liveness e la mediatizzazione della presenza
}

\author{
Vincenzo del Gaudio \\ Università degli Studi di Salerno
}

\begin{abstract}
Questo lavoro intende mostrare come la questione della liveness e, con essa la questione della presenza, possa essere posizionata piuttosto alla fine del secolo scorso. Attraverso uno sguardo mediarcheologio il lavoro intende chiarire come la liveness è figlia di negoziazioni continue tra spazio, media e corpo umano. Tale negoziazione è chiaramente oggi più profonda e complessa perché figlia di forme di mediatizzazione profonda del mondo e delle arti performative. Per questo motivo il contributo intende infine ragionare intorno al concetto di vanish liveness ovvero quelle forme di liveness digitale, prodotte da piattaforme come Instagram, Snapchat, TikTok, Clubhouse, in cui l'evento live non viene archiviato ed è fruibile, proprio come l'evento dal "vivo", soltanto da coloro che ne condividono un tempo.
\end{abstract}

This paper intends to show how the question of liveness and with it the question of presence can be positioned rather than the last century. Through a mediarcheological look, the work intends to show how liveness is, at least since the end of the 19th century, the child of continuous negotiations between space, media and the human body. This negotiation is clearly more profound and complex today because it is the result of forms of profound mediatisation of the world and the performative parts. For this reason, the contribution finally intends to reason around the concept of vanish liveness, that is, those forms of digital liveness, produced by platforms such as Instagram, Snapchat, TikTok, Clubhouse, in which the live event is not archived and is usable, just like the "live" event, only by those who share a time.

\section{Parole chiave/Key Words}

Téatrophone; digital liveness, vanish liveness; Cuocolo/Bosetti, Kepler 452; Nicola Vicidomini. 


\section{Forme della liveness e mediatizzazione}

Il dibattito sulla liveness, così come esso si è sviluppato a partire dagli anni Novanta del Novecento ${ }^{1}$, ha trovato nella pandemia e nei lockdown emanati in giro per il mondo, un solido alleato ${ }^{2}$. Improvvisamente, gli spazi dello spettacolo dal vivo sono stati interdetti, sono diventati portatori di contagio e morte mostrando non solo la fragilità del settore sul piano economico, produttivo e artistico ma anche quella legata alle difficoltà di riproporre gli eventi in altra forma. A dire il vero, già a partire dalla diffusione di massa dei media digitali, la questione della liveness era stata messa sotto una lente di ingrandimento sia dai performance studies ${ }^{3}$ che dai media studies ${ }^{4}$ per diventare un tema di contesa, spesso anche accesa, tra i due ambiti di studio. La prospettiva che viene presa in considerazione in questo scritto è di ordine sociologico e mediologico. Lo scopo principale è quello di misurare la portata dei modelli di liveness digitali a partire da due direttrici differenti e interconnesse tra loro: 1) la prima di ordine mediarcheologico ${ }^{5}$. Essa parte dal tentativo di ricostruire una soglia epistemica a partire dall'analisi dei primi dispositivi, in particolare il Théâtrophone, che hanno espanso lo spazio scenico ridefinendo i concetti di spazio e di tempo oltre a quello di presenza; 2 ) la seconda, ad essa connessa ma non necessariamente

\footnotetext{
${ }^{1}$ Per una prima ricostruzione del dibattito Crf. P. Phelan, Unmarked. The politics of performance. Routledge, London \& New York 1994; P. Auslander, Liveness. Performace in mediatized culture, Routledge, London \& New York 1999; E. Fisher- Lichte, Estetica del performativo. Una teoria del teatro e dell'arte, Carocci, Roma $2014 ; \mathrm{H}$. Blau, The Human Nature of the Bot: A Response to Philip Auslander, in «PAJ: A Journal of Performance and Art», vol. 24, n. 1, January 2002, pp. 22-24; K. Van Es, The future of live, Polity Press, Cambridge 2017.

2 Cfr. G. Boccia Artieri, L. Gemini, Per un live dei corpi a distanza, in «Doppiozero», $1^{\circ}$ maggio, 2020, https://www.doppiozero.com/materiali/un-live-dei-corpi-distanza (ultimo accesso 25/11/2021); L. Gemini, S. Brilli, F. Giuliani, Il dispositivo teatrale alla prova del Covid-19. Mediatizzazione, liveness e pubblici, in "Mediascapes Journal», n. 20, 2020, pp. 44-58; L. Gemini, Antidoto liveness. La performance dal vivo durante il Covid19 , in «Sociologia della comunicazione», n. 60, 2020, pp. 104-118.

${ }^{3}$ Crf. A. Auslander, Live and Technologically Mediated performance, in T.C. Davis (ed.) The Cambridge Companion to Performance Studies, Cambridge University press, Cambridge 2008, pp. 107-119; P. Auslander, Digital Liveness: A Historico-Philosophical Perspective, in «Journal of Performance and Art», vol. 34, n. 3, 2012, pp. 311; R. Schneider, It Seems As If... Am Dead Zombie Capitalism and Theatrical Labor, in «TDR: The Drama Review», vol. 56, n. 4, Winter 2012, pp. 150-162.

${ }^{4}$ Cfr. N. Couldry, Liveness, "reality", and the mediated habitus from television to the mobile phone, in "Communication Review», vol. 7, n. 4, 2004, pp. 353-361; L. Gemini, Liveness: le logiche mediali nella comunicazione dal vivo, in "Sociologia della comunicazione», n. 51, 2016, pp. 43-63; V. Del Gaudio, Liveness: presenza, immagine digitale e falsificazione, in A. Rabbito (a cura di), La cultura del falso. Inganni, illusioni e fake news, Meltemi, Milano 2020, pp. 437-447; A. Cristell, Liveness and Recording in the Media, Routledge, London \& New York 2012.

${ }^{5}$ Per una prima ricognizione sul concetto di mediarcheologia preliminarmente si veda: J. Parikka, Archeologia dei media. Nuove prospettive per la storia e la teoria della comunicazione, Carocci, Roma 2019; S. Arabatzis, Medienherrschaft, Medienresistenz und Medienanarchie. Archäologie der Medien und ihr neuer Gebrauch, Springer, Berlin 2017.
} 
conseguente, si basa sulla prospettiva mediologica legata alla sempre crescente mediatizzazione $^{6}$ dell'esperienza umana che coinvolge ambiti che tradizionalmente sembravano essere esterni all'orizzonte dei media e, come nel caso del teatro e delle arti performative, che spesso sono stati pensati addirittura come alternativi alla mediatizzazione. In tale prospettiva verrà presa in considerazione la nozione di "vanish liveness" intesa come modello attraverso cui riconcettualizzare il concetto di liveness a cavallo tra i media studies e i performance studies. Essa viene a determinarsi a partire dalle forme di rimediazione dalla tradizionale liveness teatrale nelle piattaforme contemporanee.

Scandagliare le forme della liveness significa quindi mettersi al centro di una rete di saperi spesso in contraddizione tra loro che si muovono in diverse direzioni. Il concetto di liveness si riferisce generalmente alla partecipazione dal vivo ad un determinato evento. Come ha notato Karin $\operatorname{Van} \mathrm{Es}^{7}$, esso è stato spesso interpretato seguendo tre posizioni differenti: una "ontologica", una "fenomenologica" e una "retorica".

Per quanto riguarda la posizione "ontologica" è evidente che gli studi sulla liveness si producono a partire dagli albori dei cosiddetti television studies. Con l'avvento della televisione e dalla sua capacità, a differenza del cinema, di trasmettere immagini dal vivo, numerosi teorici hanno pensato la liveness come tratto ontologico della televisione ${ }^{8}$, questo perché vedevano in questa, la prima forma mediale capace di connettere lo spettatore con eventi lontani in tempo reale. A dire il vero tale qualità era rintracciabile già nella radio che aveva aperto lo spazio domestico fino ad espanderlo e connetterlo con eventi che accadevano a lunga distanza ${ }^{9}$. Concentrandosi sul fatto che la liveness si produca a partire da un modello di partecipazione dello spettatore che possa condividere uno spazio fisico e un tempo, un hic et nunc, con il polo spettacolare, e pertanto possa agire attivamente sullo svolgersi dell'evento, anche gli studi sul teatro e i performance

\footnotetext{
${ }^{6}$ Sul concetto di mediatizzazione di veda preliminarmente: N. Couldry, A. Hepp, The Mediated Construction of Reality, Polty Press, Cambridge 2017; G. Boccia Artieri, Mediatizzazione e network society: un programma di ricerca, in «Sociologia della comunicazione», n. 50, 2015, pp. 60-67.

${ }^{7}$ K. Van Es, op. cit.

${ }^{8}$ Su questo punto si veda ancora Auslander, op. cit., oltre che P. Scandell, Television and the Meaning of Live: An Enquiry into the Human Situation, Polity, Malden 2014.

${ }^{9}$ Su questo punto decisive sono le intuizioni di P.W. Sewell, Television in the age of radio, Rutgers University press, London 2014. Sulla radice radiofonica della liveness fondamentale rimane il saggio di Auslander: P. Auslander, Digital Liveness: A Historico-Philosophical Perspective, in «Journal of Performance and Art», vol. 34, n. 3, 2012, pp. 3-11.
} 
studies hanno rivendicato una presunta qualità ontologica della liveness riferita alle arti performative. II tratto ontologico viene rivendicato da più forme mediali e seconda di come viene concettualizzata la liveness. È chiaro che, già ad una prima analisi, è impossibile pensare al concetto di liveness come ad una qualità ontologica per definire una singola forma mediale perché essa, trovando una determinazione in diversi media, non può essere una qualità riferibile ad un singolo medium. La questione ontologica, inoltre, contrasta con l'evidenza che la liveness si produce a partire dalle forme di partecipazione all'evento in tempo reale che, in epoca digitale, sono convergenti e multipiattaforma.

Per quanto riguarda la formulazione di natura "fenomenologica" essa si concentra sulle esperienze e le affettività delle audience. Per tale ragione essa guarda agli eventi come spazi di relazione tra utente e tecnologia, come vuole Auslander nella sua formulazione del concetto di liveness digitale del 2012, concentrandosi a partire dalle singole forme di liveness che una particolare forma mediale produce. A mio avviso però tale formulazione non tiene conto del fatto che diverse forme di liveness prodotte nelle nuove piattaforme digitali sono figlie di processi di rimediazione della liveness sviluppatesi precedentemente in altri contesi mediali. Questo implica, da un lato riconoscere la specificità delle forme di liveness prodotte all'interno di forme mediali contingenti e, dall'altro, ripensare a tale specificità a partire da quelle forme di liveness che le hanno precedute e che di fatto ne sono una rimediazione. Ad esempio, I'utilizzo delle risate registrate nelle sit-com ha come scopo quello di produrre una sorta di liveness simulata in modo da indurre lo spettatore a pensare che lo show che sta guardando stia avvenendo in quel momento davanti ai propri occhi con un pubblico dal vivo di cui ovviamente è chiamato a sentirsi parte. Questo mira a simulare la presenza di un pubblico dal vivo che condivide lo spazio fisico e il momento della produzione di uno specifico contenuto per far sentire il pubblico da casa parte integrante del processo produttivo. È evidente che tale liveness simulata ha le sue basi nell'esperienza dello spettatore teatrale e, di fatto, rappresenta una sua forma di rimediazione ${ }^{10}$.

\footnotetext{
${ }^{10}$ Su questo punto ci permettiamo di rimandare al nostro: V. Del Gaudio, Modelli di serialità teatrale: Pratiche per un approccio mediologico al teatro a partire dai modelli seriali della Tragedia Endogonidia della Sociétas Raffaello Sanzio e Ada. Una cronaca familiare di Fanny \& Alexander, in «Mediascapes Journal», n. 8, 2017, pp. $253-264$.
} 
La formulazione per la quale non esiste una liveness ma soltanto una sua "retorica" - che è la terza posizione del concetto individuata da Van Es - si basa, invece, sull'idea che sarebbero i produttori dei contenuti a creare effetti di liveness, promettendo agli utenti, una partecipazione immediata all'evento laddove ci troviamo sempre di fronte a forme complesse di mediazione. La retorica della liveness, secondo alcuni studiosi ${ }^{11}$, produce una sorta di gerarchizzazione dei media per cui la liveness diventerebbe un marcatore della presunta superiorità, sul piano dell'autenticità, della partecipazione all'evento di una forma mediale piuttosto di un'altra o di un modello di liveness piuttosto che un altro. Chiaramente, a mio avviso, questa retorica, che è spesso utilizzata dagli studi sul teatro e sulle arti performative per mostrare la presunta superiorità del patto comunicativo teatrale in quanto basato su una sorta di unicità scevra da forme di ripetizione, tende ad occultare l'evidenza che le forme di liveness si producono seguendo format specifici anche nei media sia analogici che digitali. Tale retorica si basa inoltre sulla presunta immediatezza dell'evento dal vivo rispetto dell'evento mediato. Su questo punto si concentra anche Esther Hammelburg ancorando il concetto di liveness a quelli di immediatezza e affinità ${ }^{12}$ : la liveness sarebbe immediata perché permetterebbe di connettersi agli eventi nel momento in cui accadono e sarebbe affine perché produce coinvolgimento con tali eventi ${ }^{13}$. Dal mio punto di vista tenderei a non definire la capacità dei media di connetterci con gli eventi con il termine immediatezza questo perché l'evento, per sua natura, è figlio di forme di mediazione ${ }^{14}$ spesso anche complesse e nascoste, e lo stesso evento teatrale e performativo si basa su tutta una serie di mediazioni culturali, simboliche e tecnologiche.

Seguendo queste tre formulazioni la liveness si caratterizza come un costrutto culturale basato sulla relazione tra evento, utenti e tecnologie. Tale relazione è di per sé una forma di mediazione che impedisce una qualsivoglia relazione "pura" con l'evento.

\footnotetext{
${ }^{11}$ Crf. M. White, Television and Internet Differences by Design: Rendering Liveness, Presence, and Lived Space. In «Convergence: The International Journal of Research into New Media Technologies», vol. 3, n. 12, 2006, pp. 341-55; W. Boddy, Redefining the Home Screen: Technological Convergence as Trauma and Business Plan, in D. Thorburn, $\mathrm{H}$. Jenkins (ed.), Rethinking Media Change: The Aesthetics of Television, MIT press, Cambridge 2003, pp. 191-200.

${ }^{12} \mathrm{E}$. Hammelburg, \#stemfie: reconceptualising liveness in the era of social media, in «Tijdschrift Voor Mediageschiedenis», vol. 18, n. 1, 2015, pp. 85-100.

13 Ivi, p. 87-88.

${ }^{14}$ Cfr. R. Grusin, Radical Mediation Cinema, estetica e tecnologie digitali, Pellegrini, Cosenza 2017.
} 
Questo ovviamente non significa che non ci sia alcuna differenza tra l'andare a teatro o ad un concerto e fruire dello stesso evento attraverso le piattaforme digitali ma che tale differenza è il prodotto delle diverse forme che assume la relazione di liveness e dei diversi formati che la relazione tra utente, evento e tecnologie determinano ${ }^{15}$. Per questo motivo sarei portato a dire che non esiste una forma unica di liveness quanto diversi gradienti di liveness che spesso sono interconnessi tra loro e si rimediano a vicenda. Questo significa che il concetto liveness si determina a seconda dei tipi di relazione che gli utenti intrattengono con gli eventi e le tecnologie. Ad esempio, rispetto agli eventi live prodotti dai social network, essi funzionano grazie ad un processo di rappresentazione/rimediazione della logica della diretta televisiva in epoca analogica, tale rimediazione però implica un ripensamento del medium rimediato all'interno del medium rimediante. Tale rimediazione implica, ad esempio, una diversa modalità di partecipazione agli eventi da parte del pubblico: nelle dirette prodotte nelle piattaforme social, attraverso il principio della chat e dei commenti, l'utente ha un ruolo nella determinazione dell'evento stesso, nella produzione del suo contenuto e nella forma che questo contenuto assume dove, per quanto riguarda la diretta televisiva in epoca analogica, l'attività del pubblico è irrilevante per la costituzione dell'evento. In questo caso, il rapporto con delle tecnologie e con l'evento, pur trovandoci di fronte ad un'evidente forma di rimediazione, si definisce a partire da due relazioni di liveness differenti: nel primo caso potremmo dire che l'esperienza dell'evento dal vivo che la tecnologia analogica impone, mette di fronte l'utente ad una relazione più schiacciata sull'evento e le sue forme di partecipazione ad esso sono limitate alla propria esperienza che è irrilevante per lo svolgersi dell'evento stesso. Nel secondo caso invece il rapporto con le tecnologie digitali mette l'utente in relazione biunivoca con l'evento rideterminando la relazione di liveness per generare un'esperienza e modelli di liveness specifici.

La relazione di liveness teatrale tradizionale si basa sull'hic et nunc, sulla condivisione di uno spazio e di un tempo, le forme di liveness digitale, in quanto figlie del pro-

\footnotetext{
15 Il concetto di relazione di liveness, cui sembra fare riferimento Van Es con il termine network, è in stretta connessione, con sfumature differenti, con quello di gradienti di liveness. Entrambe le formulazioni intendono la liveness come un concetto operativo che si basa sulla relazione tra utenti, tecnologie ed evento dal vivo. Crf. L. Gemini, 2016, op. cit.; K. Van Es, op. cit.
} 
cesso di mediatizzazione dell'esperienza, implicano una mediatizzazione anche dell'hic et nunc in chiave digitale.

Proprio a partire dalla relazione di liveness di tipo teatrale tradizionale bisogna tenere conto di un'altra questione: spesso la liveness viene messa in relazione con la nozione di presenza: se il concetto di liveness può essere definito a partire dai diversi gradienti di liveness, le forme che questi di volta in volta producono impongono un ripensamento anche del concetto di presenza e della sua mediatizzazione ${ }^{16}$. In particolare, $\mathrm{i}$ media digitali impongono nuove forme di presenza che si sostanziano a partire dal rapporto tra corpo e tecnologia ed evento ${ }^{17}$. Questo significa che, attraverso i media digitali è possibile pensare di partecipare ed essere presenti ad un evento in forma corporea a diversi livelli e a diversa distanza, il che significa, altresì, che le forme dell'essere presenti sono sempre legate alla messa a punto di strategie emozionali che investono il corpo a diversi livelli ${ }^{18}$. La presenza, lungi dall'essere qualcosa di unitario, legandosi a forme mediatizzate di corpo, diventa un attributo tecnologico del corpo, una sua sorta di protesi. Questo vale anche per quelle forme di presenza che a prima vista sembrano non basarsi sulle tecnologie. Ad esempio, nel teatro e nella performance, sono tante le tecnologie corporee e che investono il corpo, sia dell'attore che dello spettatore, e che sono fondamentali nel processo di produzione e consumo spettacolare. A partire da tali presupposti i media, sia analogici che digitali, dagli ultimi decenni del Novecento sino ai giorni nostri, hanno prodotto tutta una serie di strategie per produrre forme mediatizzate di presenza, il che significa forme di hic et nunc in cui il corpo è coinvolto a distanza generando forme esternalizzate di corpo. A questo proposito Mario Costa, in un testo che si propone di andare a scandagliare le radici delle forme esternalizzate di corpo, chiarisce che la prima tecnologia che crea una forma di presenza a distanza è stata il telefono:

\footnotetext{
${ }^{16}$ Crf. Gemini, S. Brilli, F. Giuliani, op. cit.; G. Boccia Artieri, L. Gemini, op. cit.

${ }^{17}$ Su questo punto interessanti sono le riflessioni di N. Coudry, Liveness, 2004, op. cit. e di A. Pizzo, Il corpo mediatizzato, in «Mimesis Journal», vol. 7, n. 1, 2018, pp. 113-125.

${ }^{18} \mathrm{Su}$ questo punto interessanti sono le riflessioni di Enrico Pitozzi nel numero dedicato alla presenza della rivista Culture Teatrali dove viene proposta la nozione di "gradazioni di presenza": E. Pitozzi, (a cura di), "Culture Teatrali. On Presence», n. 21, 2011. Per ciò che riguarda i performance studies si veda anche: G. Giannachi, N. Kaye, M. Shanks, (a cura di), Archaeologies of Presence, Routledge, London 2013.
} 
Il telefono esternalizzava la propria posizione nello spazio e nel tempo [...], il mio hic et nuc è ogni volta una porzione unica dello spazio e del tempo che mi dà consistenza e persistenza, col telefono il mio hic et nunc può trovarsi dovunque e contemporaneamente. ${ }^{19}$

Tale tecnologia viene subito sfruttata il teatro che, se fino a quel momento era stato lo spazio entro cui si determinava la presenza spettacolare, si produce in una serie di rimediazioni con al centro la tecnologia telefonica.

\section{Archeologia della presenza: Théâtrophone}

Per spiegare il modo attraverso cui la relazione di liveness sia alla base del processo di determinazione dell'esperienza dal vivo, e soprattutto il processo di rimediazione dei formati di liveness, è utile utilizzare come esempio quello del telefono che, alla fine del XIX secolo si è diffuso come tecnologia capace scorporare parti fisiche del corpo, in particolare la voce, e riposizionarle nello spazio ${ }^{20}$. Il telefono ha rappresentato una delle prime forme di mediatizzazione dell'hic et nunc e della corporeità della presenza: la posizione del corpo nello spazio diventa mobile e collocabile a distanza. II telefono, separando la voce dal corpo, ha messo in discussione il rapporto tra corpi ed eventi aprendo a forme mediatizzate di partecipazione. Il fatto che l'hic et nunc del corpo possa essere di colpo trasportabile a distanza, che parti e funzioni del nostro corpo possano essere trasportate, ha conseguenze decisive anche sul teatro. Proprio grazie alla tecnologia telefonica, il teatro vede per la prima volta messo in crisi il proprio principio comunicativo basato sulla copresenza di attore e spettatore in un unico spazio fisico in una condivisione temporale. Con la mediatizzazione dell'hic et nunc per, la prima volta, quello spazio si espandeva creando così una nuova relazione di liveness. Se alcune funzioni e percezioni del corpo potevano essere gestite a distanza questo significava che il modello di produzione e consumo del teatro poteva essere rimediato dalle nuove forme mediali che si stavano affacciando sulla scena della modernità.

\footnotetext{
${ }^{19}$ M. Costa, L'uomo fuori di sé. Alle origini della esternalizzazione: la fotografia, il fonografo e il telefono nella Parigi del XIX secolo, Meltemi, Milano 2018, p. 122.

${ }^{20}$ In questo senso ancora interessanti possono essere le riflessioni di Pierre Levy: P. Levy, Il virtuale, Raffaello Cortina, Milano 1997 e le implicazioni teatrali che esse comportano: A. Pizzo, Teatro e mondo digitale. Attori, scena e pubblico, Venezia, Marsilio 2003.
} 
Nel 1881 un dispositivo per la prima volta permette di usufruire di uno spettacolo teatrale o musicale a distanza. Questo dispositivo, che non ha ancora un nome, è presentato alla Grand Exposition d'électricité con lo scopo di illustrare il funzionamento della nuova tecnologia telefonica da parte della Société générale des téléphone per invogliare all'utilizzo del nuovo mezzo. L'ha creato Clement Adler che è stato nel 1878 I'installatore del primo telefono privato a Parigi ${ }^{21}$. La neonata tecnologia funziona a partire da 24 microfoni posti sul boccascena che permettono ai visitatori dell'esposizione di ascoltare in diretta spettacoli provenienti da tre teatri parigini: Opéra, Opéra-Comique e ThéâtreFrançais. La particolarità del Théâtrophone, che lo differenzia dal suo diretto concorrente dell'epoca, ovvero il fonografo inventato da Edison, è appunto il fatto che lo spettatore può ascoltare in diretta ciò che accade dentro ai teatri. Otto anni dopo, nel 1889, per l'esposizione Universale, presso il padiglione dell'elettricità, l'invenzione perfezionata e chiamata Théâtrophone è pronta per la commercializzazione. Oltre ai microfoni il Téatrophone funziona a partire da due cuffie stereo che, grazie alla dislocazione dei microfoni nello spazio, permettono un ascolto stereoscopico ${ }^{22}$. La nuova macchina funziona a gettoni: l'utente inserisce una moneta all'interno della macchina per ascoltare circa cinque muniti di spettacolo teatrale. II Théâtrophone è pensato per la fruizione pubblica in bar, nelle hall degli alberghi ma anche per la fruizione privata, in casa propria, attraverso un servizio di abbonamento che partirà nel 1890 con la nascita della societé du Théâtrophone. Il Théâtrophone riscuote un discreto successo, tanto che sembra che lo scrittore Marcel Proust ne fosse un entusiasta fruitore ${ }^{23}$. Le trasmissioni verranno prodotte, a fasi alterne, fino agli anni Trenta del Novecento.

Il modello del Théâtrophone si muove nella direzione della rifunzionalizzazione della relazione di liveness che per la prima volta, almeno per ciò che riguarda il teatro e le arti performative, si apre a un nuovo modello spaziale che si fonda sulla relazione tra

\footnotetext{
${ }^{21}$ Per una prima ricognizione storica sul Théâtrophone Cfr. M. Costa, op. cit., D. Laster, Plendeurs et misères du "Théâtrophone", in «Romantisme», n. 41, 1987, pp. 74-78; C. Bertho-Lavenir, Innovation technique et société du spectacle: le théâtrophone à l'Exposition de 1889, in «Le Mouvement social», n. 149, pp. 59-59.

${ }^{22}$ Crf. G. Pisano, The Théâtrophone, an Anachronistic Hybrid Experiment or One of the First Immobile Traveler Devices? In A. Gaudreault, N. Dulac, S. Hidalgo (a cura di), A Companion to Early Cinema, Blackwell Pub, Hoboken 2012, pp. 80-98.

${ }^{23}$ Crf. K. Von Hagen, Devant le téléphone: Sinn und Sinnlichkeit oder das théâtrophone bei Proust, in U. Felden, V. Roloff (a cura di), Die Korrespondenz der Sinne. Wahrnehmungsästhetische und intermediale Aspekte im Werk von Proust, Brill, Berlin 2008, pp. 215-232.
} 
spazio e tecnologie. II Théâtrophone permette allo spettatore di acquistare una nuova centralità nella relazione di liveness aprendo a forme mediatizzate di hic et nunc che si basano su diversi gradienti di liveness. Per la prima volta, e qualche anno prima della diffusione di massa della radio, è possibile ascoltare in diretta la voce dell'attore svincolata dal corpo aprendo a spazi di significazione a distanza che si modellano sul principio della percezione spazializzata che definisce la nuova tecnologia teatro-telefonica. II Théâtrophone rappresenta una forma mediatizzata del patto teatrale attraverso cui viene detronizzato l'hic et nunc come indice per determinare ontologicamente cosa sia teatro da ciò che non lo sia e mostrare l'evidente apertura dello spazio fisico a forme di mediatizzazione sempre più complesse che tendono ad espanderlo. Separando il punto di ascolto dalla produzione del suono il Théâtrophone rompe il principio della copresenza fisica di produzione e consumo dell'informazione mettendo in rilievo il fatto che tale principio è di natura più formale che fenomenica e funziona a partire da contingenze socio-tecno-mediali e cultuali che sono figlie di ciò che una determinata cultura e determinate condizioni storiche concettualizzano come esperienza live. Le forme di presenza a distanza prodotte dai nascenti media acustici della modernità mostrano come il teatro rimedi e rifunzionalizzi la nascente tecnologia telefonica piegandola alla propria logica spettacolare. Interessante, infine, è anche il modello di fruizione del Théâtrophone, esso si basa su di una doppia logica che ha basi profondamente teatrali: 1) il dispositivo tecno-teatrale apre a forme di fruizione che oggi definiremmo live on demand: ovvero l'ascoltatore, attraverso l'uso della monetine, può scegliere i pezzi di spettacolo a cui collegarsi ${ }^{24}$; questo principio ha anche l'effetto di aprire il teatro, inteso come spazio fisico, allo spazio urbano delle nascenti metropoli moderna e ad un diverso modello di fruizione collettiva; 2 ) Oltre alla fruizione pubblica, legata al fatto che il Théâtrophone era installato in alberghi bar e altri luoghi pubblici, si fa largo anche una fruizione domestica. Essa crea un doppio effetto: da un lato anticipa la fruizione domestica dei media successivi, tra tutti la radio e successivamente la televisione, dall'altro risemantizza la relazione sociale tra spazio dell'intrattenimento e del tempo

\footnotetext{
${ }^{24}$ Molto spesso gli utenti si collegavano ad una certa ora piuttosto che ad un'altra per ascoltare un determinato monologo o una determinata scena piuttosto che un'altra.
} 
libero, che proprio in quegli anni si apre a modelli mondani, e spazio della casa inteso come spazio privato che si differenzia della sospensione del quotidiano tipica degli spazi festivi riservati allo svago. Il gradiente di liveness creato dal dispositivo telefonico teatrale apre a modelli di fruizione che poi saranno centrali nella determinazione dei consumi delle forme mediali successive sia analogiche che digitali. Mostrando come, in realtà, la deflagrazione dello spazio scenico non sia una conseguenza dell'avvento dei media digitali, ma piuttosto come essa si basi su forme di mediatizzazione a medio e lungo raggio, il Théâtrophone può essere considerato come una forma antesignana delle forme di liveness prodotte dai media e prodotte per tutto il secolo scorso e per il primo ventennio del nuovo. Questo chiaramente non significa che tutte le forme mediatizzate di liveness successive siano derivanti della tecnologia telefonico-teatrale (essa ha forme di rimediazione proprie), piuttosto, che la presunta unità di spazio e di tempo che si situerebbe alla base del medium teatrale ${ }^{25}$ è di natura culturale e sociale.

\section{Théâtrophone remediation: il teatro al telefono}

Il Théâtrophone apre le porte del teatro, ne amplifica lo spazio sino a portarlo all'interno delle case, dei bar, delle hall degli alberghi. Facendo questo, ridefinisce la relazione di liveness. La liveness intesa nella sua forma tradizionalmente teatrale, come copresenza di attore e spettatore che condividono uno spazio e un tempo, può essere rimediata dalle forme mediali della modernità e tale rimediazione definisce nuovi formati di liveness basati su rifunzionalizzazioni della relazione di liveness. In questo senso la rimediazione, intesa come la rappresentazione delle logiche di un medium in un altro, diventa il motore che determina nuovi formati della relazione di liveness. Il formato di liveness alla base del Théâtrophone, ad esempio, è stato rimediato dalla radio, dalla televisione e, infine, dai media digitali. La radio ha rifunzionalizzato la relazione di liveness attraverso la possibilità dell'ascolto dal vivo di concerti e drammi trasmessi sia dall'interno dei teatri che dagli studi radiofonici. Questo modello di drammaturgia sono-

\footnotetext{
${ }^{25}$ Sul teatro come forma mediale ci permettiamo di rimandare al nostro: V. De Gaudio, Théatron. Verso una mediologia del teatro e della performance, Meltemi, Milano 2020 oltre che a: F. Deriu, II Teatro È Un Medium? Questioni E Risposte In Prospettiva "Mediologica", in "Mantichora», n. 5, dicembre 2015, pp. 59-65; D. Sacco, Teatro come medium. Sulla riproducibilità a partire da Walter Benjamin e Samuel Weber, in «Dradek», vol. III, n. 1-2, 2017, pp. 87-109.
} 
$\mathrm{ra}^{26}$, e le sue forme rimediate, aprono alla configurazione di diversi gradienti di liveness. La questione della rimediazione della relazione di liveness si inserisce all'interno della possibilità dei media in generale di ripensare la relazione tra utente, tecnologie ed evento per generare formati differenti. Questo significa che, al centro di tale rimediazione troviamo l'esperienza dell'utente che è, di volta in volta, alla base della relazione di liveness. Quest'ultima si situa nel corpo dell'utente ridefinendo l'esperienza che questo fa degli eventi dal vivo. La rimediazione delle forme della relazione di liveness inoltre implica il fatto che, molto spesso, le radici dei formati di liveness digitale posso essere rintracciate all'interno delle pratiche analogiche precedenti e che nel processo rimediativo la relazione di liveness si adatta la medium rimediante per definire nuovi formati live. Per spiegare questo processo, è possibile mostrare alcuni esempi in cui il telefono, sia in chiave analogica che digitale, è stato rimediato dal dispositivo teatrale. I tre esempi che abbiamo scelto sono stati tutti prodotti in Italia durante il primo lockdown della pandemia da Covid-19 e sono: 1) Theatre on a line della compagnia Cuocolo/Bosetti; 2) Favole al telefono prodotto della compagnia Campsirago Residenza; 3) Consegne // una performance da coprifuoco della compagnia Kepler 452. La scelta è ricaduta su due spettacoli che utilizzano il telefono nella sua forma evidentemente analogica, pur utilizzando la tecnologia digitale, e un altro in cui il processo di rimediazione avviene sulla base della rimediazione di tale tecnologia in chiave digitale. Questi tre esempi, lungi dall'essere esaustivi, ci mostrano tre diverse modalità di darsi del processo di rimediazione e soprattutto mostrano come alcune esperienze teatrali contemporanee possano essere lette alla luce delle tecnologie teatrali-telefoniche fine ottocentesche.

Lo spettacolo Theatre on a line è stato prodotto dalla compagnia Cuocolo/Bosetti, dal teatro della Tosse e dal teatro di Dioniso per la ripartenza dopo il primo lockdown ed è andato in scena quando i teatri erano ancora chiusi. La compagnia Cuocolo/Bosetti non è nuova ad operazioni di teatralizzazione, anche attraverso i media dell'ascolto, di spazi pubblici e privati, come strade, città, piazze e case ${ }^{27}$. Lo spettacolo funziona a partire da un dispositivo telefonico: una volta che lo spettatore ha comprato il biglietto gli viene da-

\footnotetext{
${ }^{26}$ Crf. V. Valentini, Drammaturgie Sonore, Bulzoni, Roma 2012; R. Sacchettini, La radiofonica arte invisibile. II radiodramma italiano prima della televisione, Titivillus, Pisa 2011; A.I. De Benedictis, Radiodramma e arte radiofonica. Storia e funzioni della musica per radio in Italia, EDT, Torino 2005.

27 Sul teatro della compagnia Cuocolo/Bosetti si veda: L. Bevione (a cura di), Interior sites project. II teatro di Cuocolo/Bosetti. IRAA Theatre, Titivillus, Pisa 2017.
} 
to un numero di telefono da chiamare ad un orario stabilito. Una volta chiamato, all'altro capo della cornetta Roberta Bosetti risponde e inizia una conversazione: lo spettatore/ascoltatore può decidere di intervenire e rispondere alle domande che gli vengono poste dall'attrice o di ascoltare il monologo in silenzio. Nel caso in cui decidesse di intervenire diventa parte integrante della narrazione, così Renato Cuocolo e Roberta Bosetti spiegano il meccanismo di fruizione alla base di Theatre on a line:

\begin{abstract}
Al centro della nostra riflessione c'è il rapporto con lo spettatore. Un teatro in cui I'aspetto partecipativo, interattivo viene esaltato. Dove lo spettatore/ospite è libero di muoversi, di esplorare lo spazio, di adottare vari punti di vista: è privo, insomma, del "posto assegnato". Un teatro dove gli spettatori diventino partecipi dell'opera: si pongano in rapporto reciproco generando tra loro relazioni fisiche di prossimità, $\mathrm{o}$ in questo caso di distanziamento, animando il lavoro da molteplici angolature. Ci sono molti modi di immaginare il posto del teatro. Uno è costruito di mattoni e cemento, con un sipario rosso e una serie di posti numerati; un altro è un posto immaginario pieno di giustapposizioni politiche e incontri psicologici. Non dico di abbandonare i teatri a cui, anche se non li uso, sono tanto legato, ma d'intraprendere contemporaneamente un viaggio teatrale, al di là delle abitudini, che incorpori i temi mitici dell'interrogarsi, del ricercare, che serva come mappa di desideri, sogni, paure. ${ }^{28}$
\end{abstract}

Lo spettacolo, dunque, parte dell'idea di indagare il distanziamento attraverso il dispositivo telefonico. La spazio scenico si dilata ridefinendo la relazione di liveness che si basa su un modello di fruizione che invece di andare da uno ai molti, dall'attore agli spettatori, si definisce a partire da una relazione più intima che va da uno $\mathrm{ad} \mathrm{uno}^{29}$, dall'attore allo spettatore. II gradiente di liveness telefonico si basa sulla messa in relazione come forma di teatro e sulla produzione di un hic et nunc mediatizzato che si innerva sulla condivisione di un tempo tra attore e spettatore e di uno spazio dato mediaticamente. La relazione di liveness di Theatre on a line, rimediando la relazione di liveness del Théâtrophone, produce effetti di presenza tangibili che sono linfa narrativa per lo spettacolo; essa, inoltre mettendo in relazione due forme di spazio, quello fisico delle case dell'attrice e dello spettatore, e quello mediale, mette in gioco realtà

\footnotetext{
${ }^{28}$ R. Cuocolo, R. Bosetti, Theatre on a line, http://www.teatrodidioniso.it/in-evidenza/theatre-on-a-line-dicuocolo-bosetti-per-il-teatro-della-tosse/ (ultimo accesso 30/11/2021).

${ }^{29}$ Su questo punto, oltre alla già citata L. Gemini, op. cit., 2020 si veda anche L. Jervis, Immersive Embodiment. Theatres of Mislocalized Sensation, Palgrave Macmillan, London \& New York 2019; E. Nedelkopoulou, Attention oplease! Changing modes of engagement in device-enabled one-to-one performance encounters, in "Contemporary Theatre Review», vol. 27, n. 3, 2017, pp. 353-365.
} 
miste $^{30}$ che sono sempre state al centro del lavoro della compagnia. II gradiente di liveness di Theatre on a line si costituisce a partire dalla possibilità di pensare al teatro non esclusivamente come un luogo fisico ma come «un posto immaginario pieno di giustapposizioni politiche e incontri psicologici» il che significa pensarlo come luogo della relazione e della mediazione come fulcro della relazione stessa. A differenza di ciò che avveniva per il Théâtrophone, in cui lo spettatore non poteva intervenire allo spettacolo, la performance di Cuocolo/Bosetti ridetermina la relazione di liveness nello spazio telefonico innervandola su di una dimensione di liveness interattiva. A prima vista, questo modello di liveness è di natura strettamente analogica e si basa sulle funzioni che il telefono ha assunto nel panorama mediale da più di cento anni. La cosa interessante però è che la tecnologia digitale permette alla performance di riposizionarsi su di un secondo piano, su quelle forme di restage che Patrik Lonergan ha identificato come forme di liveness "indiretta" ${ }^{31}$, ovvero quelle forme di rimessa in scena di una performance primaria che avvengono all'interno dello spazio performativo dei social network. La performance di Cuocolo/Bosetti tiene insieme funzioni della tecnologia tipiche dell'esperienza telefonica digitale - la dislocazione spaziale generata dai telefoni cellulari ad esempio - con la tradizionale forma analogica che produce la possibilità di trasmettere la voce a distanza.

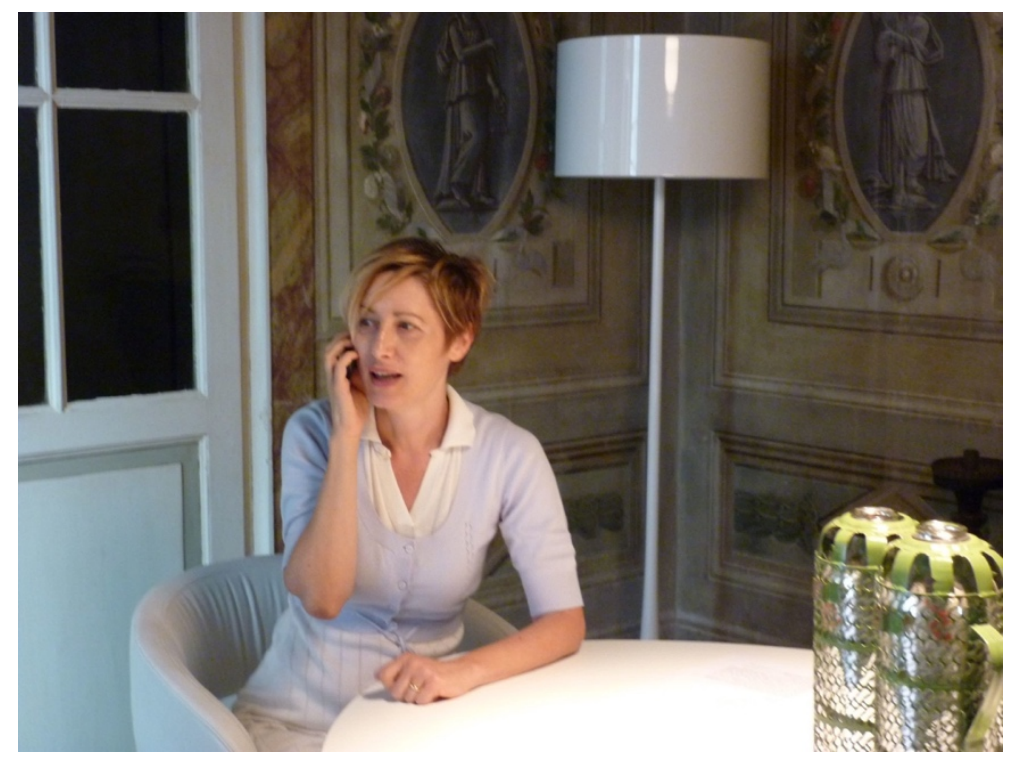

Fig. 01. Roberta Bosetti in Theatre on a line. Foto Cuocolo/Bosetti

\footnotetext{
${ }^{30}$ Sul concetto di mixed reality si veda preliminarmente: S. Banford, G. Giannanchi, Performing Mixed Reality, MIT press, Cambridge 2011; A.M. Monteverdi, Leggere lo spettacolo multimediale, Dino Audino, Roma 2020; V. Del Gaudio, Interactive Tools Performance: Blast Theory between Media Theory, Performance Studies and Social Research, in «Comunicazioni Sociali», n. 1, 2021, pp. 79-86.
}

${ }^{31}$ P. Lonergan, Theatre \& Social Media, Palgrave Macmillan, London 2013. 


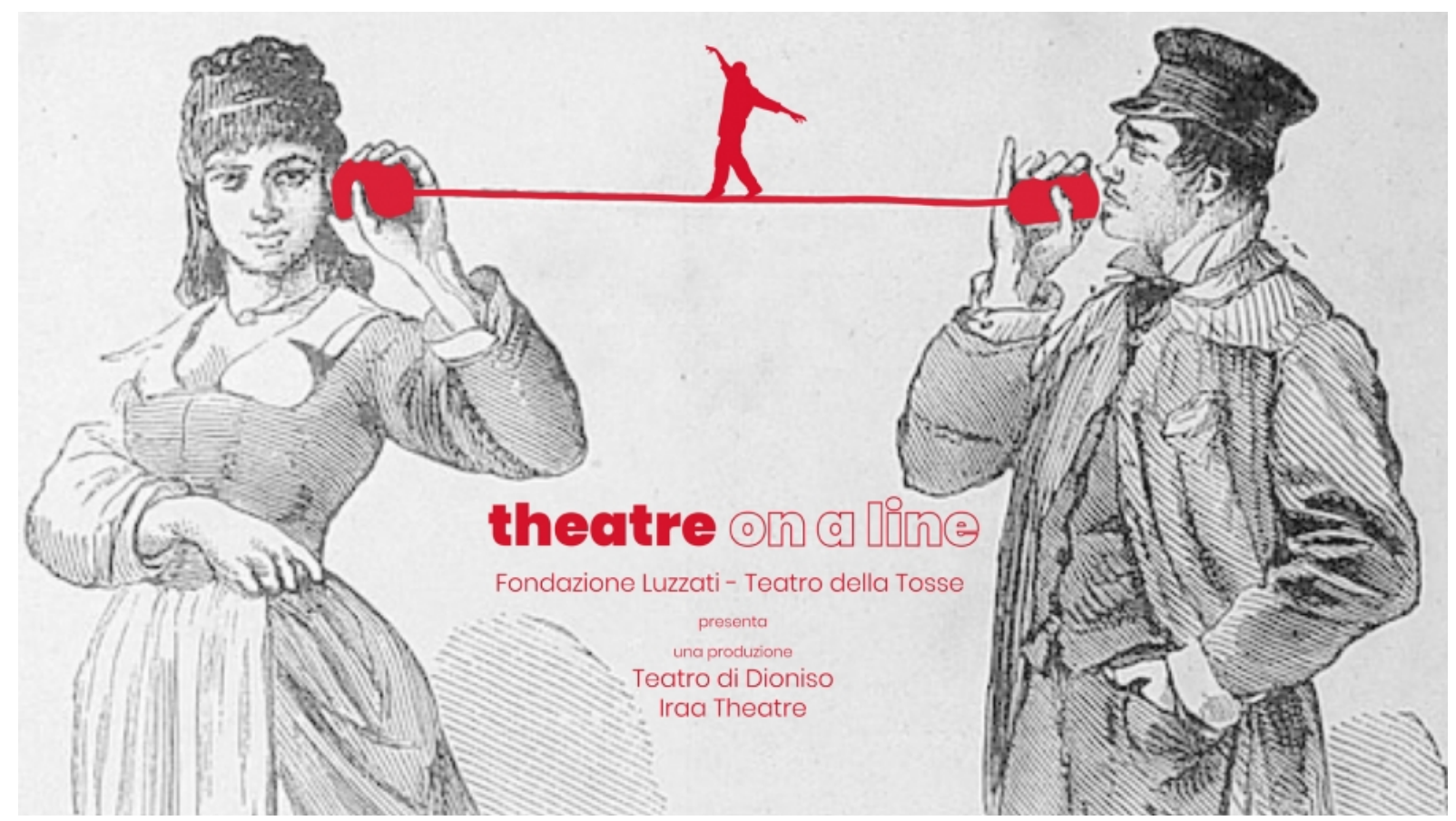

Fig. 02. Locandina di Theatre on a line

Il secondo esempio è quello dello spettacolo Favole al telefono, anch'esso prodotto durante il primo lockdown dalla compagnia Campsirago Residenza. Per la chiusura dei teatri la compagnia immagina di leggere al telefono le favole di Gianni Rodari intitolate appunto Favole al telefono. Il modello di fruizione è simile a quello dello spettacolo di Cuocolo/Bosetti: lo spettatore/ascoltatore prenota una lettura per un determinato orario dando un numero di telefono sul quale verrà contattato dall'attore. II modello di Campsirago Residenza crea una deflagrazione dello spazio scenico basata su una relazione di liveness intima, in cui lo spettatore viene messo al centro del processo comunicativo a partire dal fatto che ogni singola lettura è personalizzata e pensata per un unico spettatore. È subito chiaro che tale esperienza produce una sorta di continuità tra il modello di fruizione telefonica analogica e quella digitale. Questo modello crea almeno due dei livelli di liveness teatrale e performativa rintracciati da Peggy Phelan: 1) il fatto che la performance si definisca a partire dalla sua evanescenza, dal fatto che essa si consuma mentre la si produce; 2) il fatto che essa è ancorata al presente ${ }^{32}$.

Questo dimostra che il modello di relazione di liveness digitale, in determinati casi, pur rispettando i dettami del modello phelaniano si basa su uno spazio di mediazione

\footnotetext{
${ }^{32}$ P. Phelan, op. cit., p. 146.
} 
e, lungi dall'essere di natura ontologica, si basa su condizioni tecnologiche, culturali e sociali contingenti. Inoltre, oggi il telefono ha funzioni enormemente più complesse rispetto al dispositivo di fine Ottocento: è il fulcro della convergenza digitale e quindi i modelli di rimediazione telefonica dello spazio scenico non possono essere limitati alla voce. D'altronde nel 2007, proprio lavorando ancora sul testo di Rodari, Carlo Presotto e Titino Carrara mettevano in scena Favole al (video)telefono in cui, grazie alle videoscenografie di Giacomo Verde, e all'allora innovativa tecnologia della videochiamata, si mostrava l'evoluzione del telefono da uno strumento fatto per l'ascolto a uno strumento multimediale che andava via via includendo gli altri media.

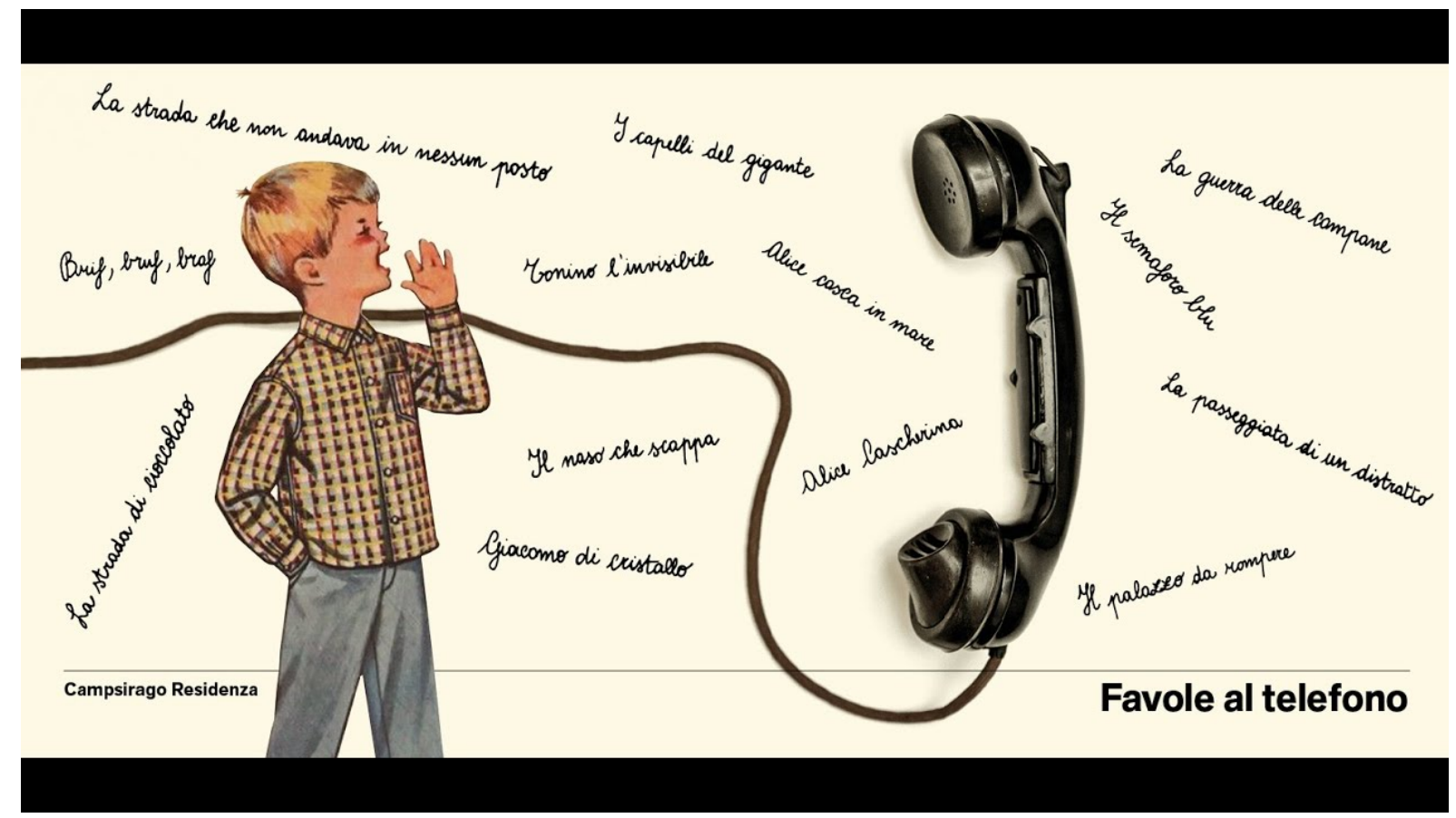

Fig. 03. Locandina di Favole al telefono

Il terzo esempio parte proprio dalla constatazione del cambiamento tecnomediale dei dispositivi telefonici. A partire dalla multifunzionalità dei nuovi smartphone, nella performance Consegne // una performance da coprifuoco, la compagnia bolognese Kepler 452 crea un gradiente di liveness telefonica mobile. La performance è prodotta nei giorni più duri del lockdown durante i quali la compagnia, come forma di reazione alla chiusura dei teatri, decide fare di un attore un rider, una delle poche figure che poteva girare liberamente nelle strade deserte, per produrre una performance a domicilio. La cosa particolare è che il rider/attore ha sul casco una telecamera e l'utente/spettatore, 
dopo aver ordinato a qualcosa a domicilio tramite una app, può seguire il tragitto del rider tramite la piattaforma digitale Zoom finché questo non giunge a destinazione dove si intrattiene con lo spettatore in una performance teatrale uno ad uno. Così la compagnia spiega il significato della performance:

\begin{abstract}
Abbiamo pensato a quali attività possono continuare a esistere in questo contesto fosco, doloroso, angosciante e la figura che si è immediatamente formata nella nostra immaginazione è quella del corriere, del rider. Quelli che non hanno smesso mai di lavorare, nemmeno nella fase più dura del lockdown. Abbiamo dunque pensato di far travestire un attore da rider, o meglio, di farlo diventare a tutti gli effetti un rider. $\mathrm{E}$ di effettuare delle consegne. Quella tra rider e destinatario è la relazione più impersonale e distaccata che si possa immaginare, e questo è il motivo per cui può resistere ad ogni chiusura. Abbiamo pensato di impiegarla e di forzarla dall'interno, per costruire un rapporto di intimità, esclusività, confidenza. Per tutta la durata del viaggio attraverso la città deserta, il corriere sarà collegato col destinatario della sua consegna, offrendo una prospettiva sul vuoto urbano altrimenti impossibile. Fino all'inevitabile suono del campanello. In un momento in cui imperversa la riflessione su quali attività siano essenziali e quali non lo siano, abbiamo desiderato domandarci che cosa sia essenziale per noi e cosa possa esserlo per gli spettatori, attraverso una domanda molto concreta: che cosa ti piacerebbe ricevere a casa tua, in questo momento ${ }^{33}$
\end{abstract}

Lo spettacolo esplicita un modello di fruizione uno ad uno e si nutre sul piano narrativo e drammaturgico dell'interazione tra spettatore e attore. Sul piano della liveness, se da un lato ci troviamo di fronte ad una forma di rimediazione del processo di produzione e consumo della scena telefonica, determinato dall'uso delle app telefoniche e degli strumenti tecnologici dei dispositivi di telefonia mobile dall'altro, la relazione di liveness si ancora all'utilizzo delle piattaforme per la comunicazione che producono esse stesse effetti di liveness. Sul piano delle relazioni di liveness la performance conduce una doppia riflessione: 1) per tutta la prima parte essa si basa su una relazione di liveness che ha nei media digitali e nella contemporaneità che essi producono il suo fulcro. Questa relazione si basa sulla possibilità di condividere un tempo, una sorta di nowness ${ }^{34}$ che è legata alla mediatizzazione del nunc piuttosto che dell'hic, dell'ora piuttosto che del qui; 2) nella seconda parte si sviluppa una liveness di tipo più classicamente teatrale, cioè legata alla condivisione di uno spazio fisico e di un tempo da parte dell'attore e dello spettatore. Lo scarto tra questi due formati di liveness viene colmato dal meccanismo narrativo

\footnotetext{
${ }^{33}$ Kepler 452, Consegne // una performance da coprifuoco, https://kepler452.it/CONSEGNE (ultimo accesso 30/11/2021).

${ }^{34}$ Sul concetto di nowness si vedano i già citati lavori di Laura Gemini.
} 
che mostra come la relazione di liveness, lungi dall'essere unica - ad ogni evento è associata un'unica relazione di liveness - può variare e assumere forme diverse e attingere a formati differenti sempre stesso evento performativo. Questo significa che in un determinato evento possono coesistere anche più relazioni di liveness differenti che implicano diverse forme di liveness digitale che convivono e, a seconda dell'esperienza dello spettatore, definiscono cosa possa essere definito come live.
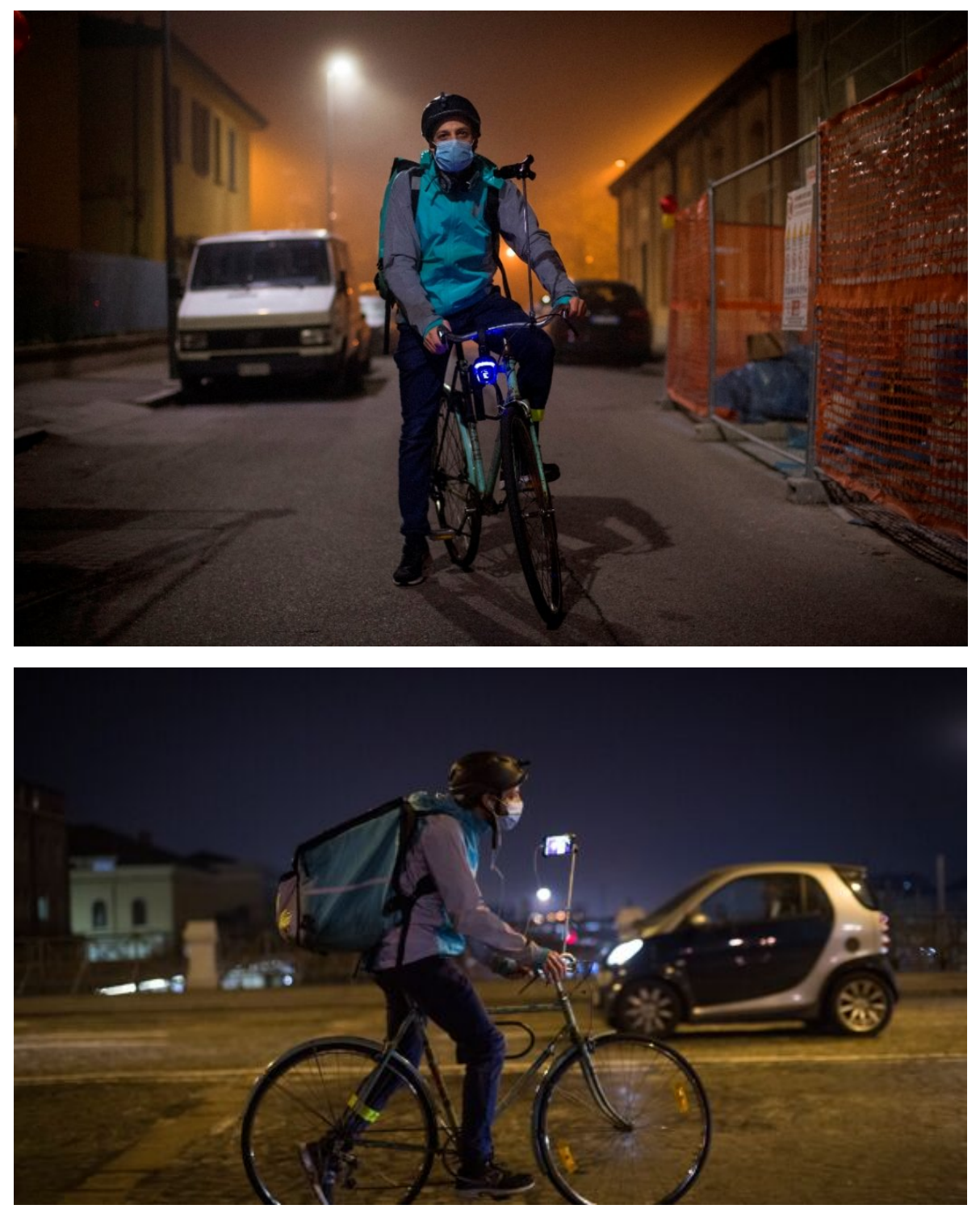

Figg. 04-05. Consegne di Kepler 452. Foto: Davide Spina

\section{Vanish Liveness}

Ho più volte accennato alla retorica della liveness come spazio di produzione dell'ideologia per la quale il teatro e le arti performative siano detentori di una forma di 
liveness pura rispetto alle forme di liveness prodotte dai media. Tale retorica si basa sulla posizione di Peggy Phelan:

L'unica vita della performance è nel presente. La performance non può essere salvata, registrata, documentata o altrimenti partecipare alla circolazione delle rappresentazioni: una volta che lo fa, diventa qualcosa di diverso dalla performance. Nella misura in cui la performance tenta di entrare nell'economia della riproduzione, tradisce e diminuisce la promessa della sua stessa ontologia. ${ }^{35}$

È evidente come alcune forme di spettacolo legate all'orizzonte dei media digitali rispettino alcune delle caratteristiche di base di quella che potremmo definire la forma tradizionale della relazione di liveness. Per certi versi questo vale anche per la performance telefoniche e, in linea di principio, per tutte quelle forme di performance che vengono prodotte nelle reti digitali attraverso le piattaforme di meeting come Zoom, Google Meet, Skype che, evidentemente, a loro volta sono forme rimediate del processo di mediatizzazione dell'hic et nunc prodotto dalla tecnologia telefonica. Definisco la relazione di liveness alla base di queste performance come "vanish liveness" intendendo con questo termine quelle forme di rimediazione digitale dell'esperienza della relazione di liveness teatrale tradizionale con un diverso gradiente. Esse si basano sul doppio principio mostrato da Peggy Phelan, ovvero l'impermanenza, cioè il fatto che la performance si consumi nel suo farsi e l'idea ad essa collegata che la performance viva solo nel presente. Queste due caratteristiche possono essere utilizzate per la comprensione di alcune performance sia artistiche che culturali prodotte dell'orizzonte dei social network e della vita nelle reti. In prima istanza è utile richiamare alla coppia concettuale coniata da Bree Hadley e Patrik Lonergan tra una performance digitale e la sua versione $\operatorname{archiviata}^{36}$. Tale differenza si riferisce alle performance che effettivamente avvengo in real time rispetto alle forme archiviate di quelle stesse performance. I modelli di archiviazione differiscono da piattaforma a piattaforma: per ciò che concerne Facebook le forme di archiviazione riproducono la forma live attraverso non solo la riproduzione audio-video ma anche attraverso i commenti. Questi appaiono nello stesso istante in cui sono stati scritti durante la diretta in modo da dare l'impressione all'utente che sta guardando la performance registrata di essere anch'egli in

\footnotetext{
${ }^{35}$ P. Phelan, op. cit., p. 146 [traduzione nostra].

${ }^{36}$ B. Hadley, Theatre, social media and meaning making, Routledge, London \& New York, 2017; P. Lonergan, op. cit.
} 
diretta. Questo produce una sorta di liveness simulata che ha la sua radice nella possibilità che l'utente ha di sentirsi parte della produzione del processo di comunicazione mentre esso è in atto. Per quanto riguarda YouTube, una volta finita la diretta, la visione registrata della performance non differisce da un evento registrato, da uno dei tanti video caricati sulla piattaforma, mostrandone il valore archiviale e azzerando di fatto la liveness. Instagram, TikTok, Snapchat invece basano la loro relazione di liveness sul fatto che essa, appunto, rimedia l'impermanenza dell'evento teatrale e performativo producendo così performance che vivono nel presente. Per Instagram il processo non vale esclusivamente per le live quanto anche per le stories, la cui permanenza è legata un tempo limitato, ovvero 24 ore, ma la loro relazione di liveness è tutta piegata sulla fruizione piuttosto che sulla produzione del contenuto. Inoltre, a differenza di TikTok e di Snapchat, Instagram permette una forma di archiviazione, la IG TV, ma tale archiviazione è spesso di tipo parziale. Le piattaforme di live chat meeting come Zoom, Google Meet, Skype, permettono l'archiviazione solo su richiesta dell'utenza, ovvero, di default gli eventi che avvengono via Zoom o via Skype non vengono registrati a meno che l'organizzatore non ne chieda la registrazione. Queste forme periture di live tendono a sparire producendo una vera e propria "Vanish liveness" in cui l'evento diventa reperibile come nella relazione di liveness teatrale tradizionale tanto da rimediarne anche le logiche di ritualità e di costruzione comunitaria. Ovviamente la "Vanish liveness" non è prerogativa dei media digitali, una sua prima formulazione la troviamo appunto nel telefono come modello di mediazione dell'hic et nunc che si basa sulla deperibilità dell'evento e, per ciò che riguarda il teatro, nel dispositivo telefonico-teatrale dato dal Théâtrophone.

In tale prospettiva le performance digitali creano nuovi formati di liveness alla cui base c'è una diversa conformazione della relazione di liveness. Quindi piuttosto che parlare di liveness digitale, tenderei a parlare di gradienti di liveness digitale mettendo un accento sulla pluralità delle possibilità di configurare la relazione di liveness che i media digitali implicano. Un esempio di tale logica può essere ritrovato nella serie di performance digitali che l'attore Nicola Vicidomini ha prodotto su Instagram nei mesi di ottobre e novembre del 2021. Le performance hanno il loro fulcro nella relazione di liveness tra il performer e gli utenti. L'attore, attraverso le stories e l'utilizzo dell'adesivo digitale per interagire con 
il pubblico mette in scena cinque diverse narrazioni performative interconnesse tra loro: 1) Rovina il nome in cui si rovinano nomi su richiesta; 2) Parla con Floyd; 3) I mestieri del jazz; 4) L'affare Bolsciolti; 5) Dispetti alla società con don Giuseppe. In Rovina il nome il pubblico, attraverso l'utilizzo dell'adesivo che generalmente viene usato per rispondere alle domande degli utenti, può chiedere all'attore di rovinare il proprio nome o un nome di fantasia; in questo modo l'attore mette in scena una serie di personaggi bizzarri che raccontano storie paradossali rispetto ai nomi che gli vengono inviati e di cui crea identità e relazioni sociali. Nella seconda narrazione, grazie ad un filtro che trasforma l'attore un cartone animato, prende vita il personaggio di Floyd, il quale risponde alle domande degli utenti con evidente ingenuità in modo unpolitically correct. II personaggio di Floyd viene intervallato da un prete feticista chiamato Don Giuseppe che mette sotto la lente d'ingrandimento

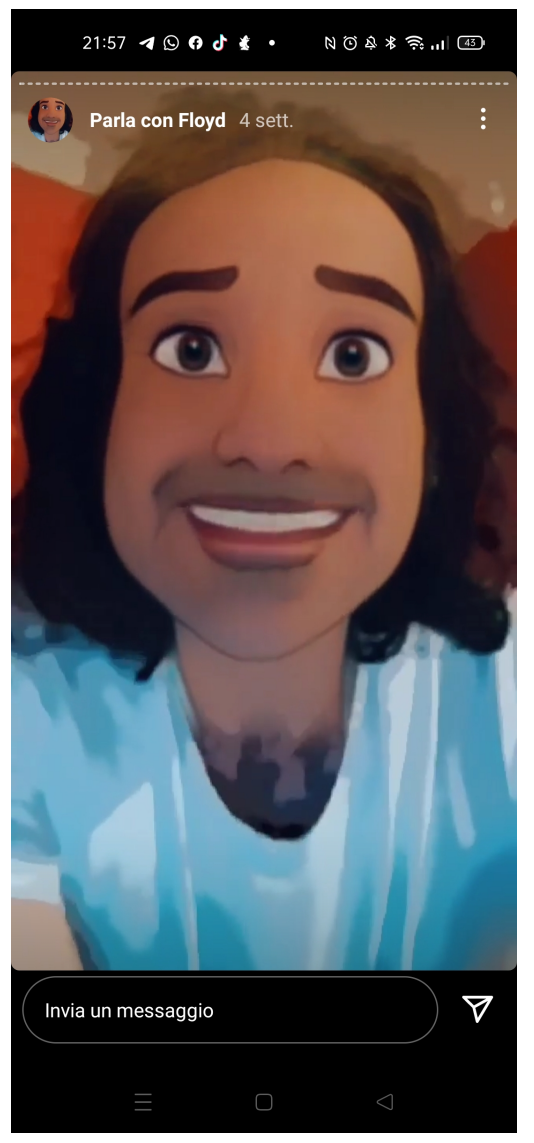

Fig. 06. Nicola Vicidomini interpreta Floyd. Foto: Baracca Vicidomini l'intera costruzione ideologica del linguaggio politicamente corretto. In I mestieri del jazz l'attore chiede agli utenti quale mestiere preferiscono a partire dai quali costruire performance musicali e narrazioni in salsa Jazz. Ne L'affare Bolsciolti, un personaggio racconta un fatto di cronaca: l'omicidio dell'onorevole Bosciolti che è stato al centro anche delle narrazioni precedenti oltre ad essere una delle narrazioni inedite del format televisivo Prublemi di Matematica. Infine, in Dispetti alla società con don Giuseppe il performer, in una sorta di posta del cuore grottesca, recupera il personaggio di don Giuseppe per prodursi in consigli di vendetta surreali contro la società. Sfruttando a pieno le logiche di produzione e consumo di Instagram l'attore produce una drammaturgia collettiva basata sul principio della "Vanish liveness". Tale "Vanish liveness" si articola secondo un doppio movimento: da un lato la liveness degli utenti che, in tempo reale, oltre a proporre i contenuti commentano le stories costruendo una relazione di liveness intima con l'attore via direct e, dall'altro produce performance deperibili che scompaiono dopo 
24 ore. Tali narrazioni hanno un'evidente natura intermediale in quanto le performance digitali diventano parte integrante della narrazione teatrale aggiungendo così al progetto un evento basato sulla tradizionale liveness teatrale.

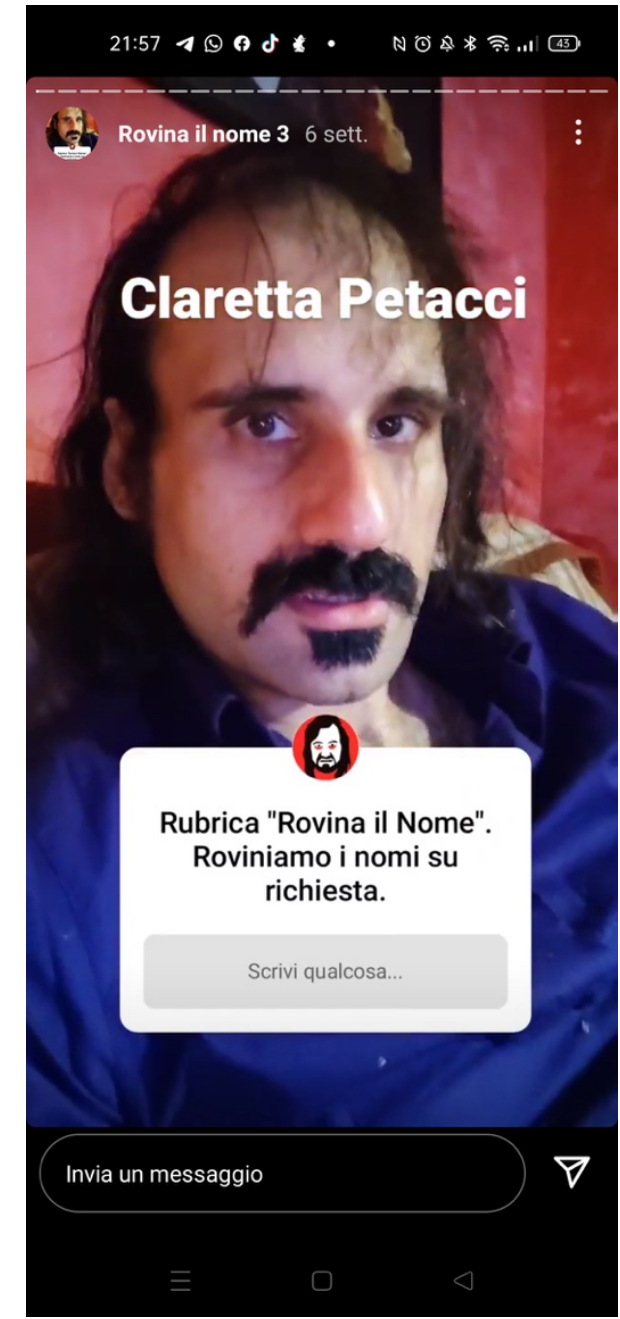

Fig. 07. Rovina il nome di Nicola Vicidomini. Foto: Baracca Vicidomini

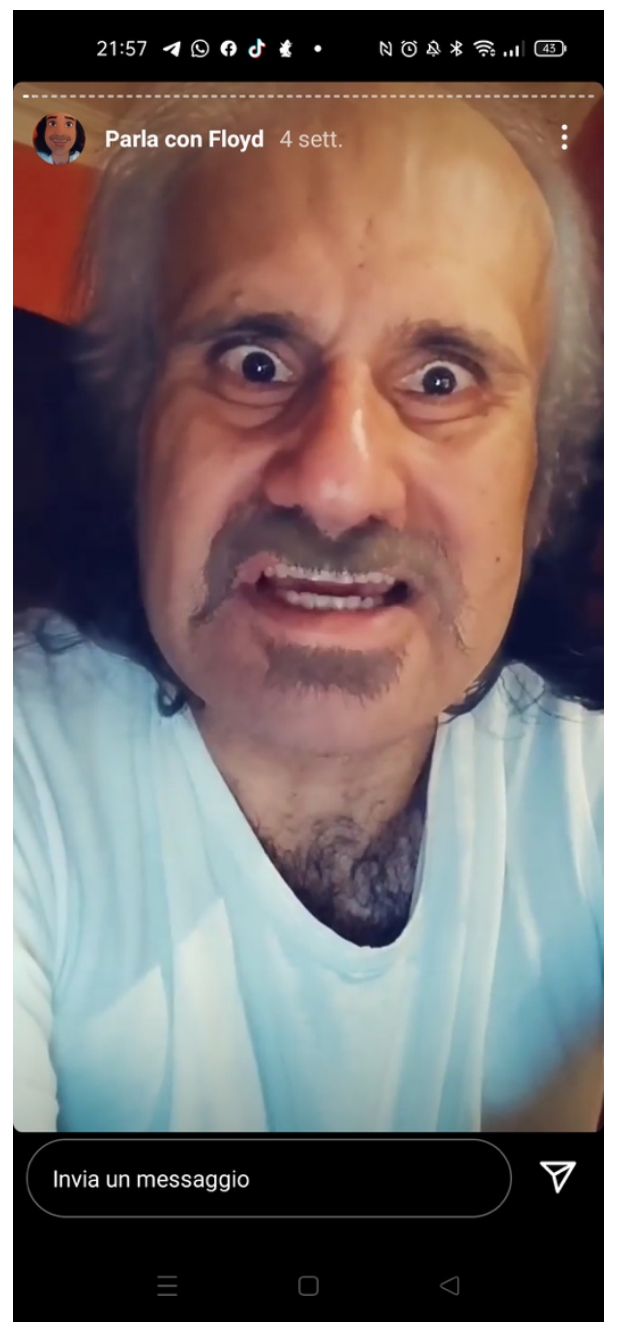

Fig. 08. Nicola Vicidomini interpreta Don Giuseppe. Foto: Baracca Vicidomini

\section{Conclusioni}

La questione della liveness come essa si è andata sviluppando all'interno del dibattito contemporaneo diventa decisiva per la messa a punto della dimensione live di qualsiasi evento. Ciò che emerge a mio avviso può essere schematizzato in questo modo: 1) La liveness è un costrutto culturale, sociale e tecnologico che, lungi dall'essere ontologicamente dato è figlio di indicatori storico-sociali che sono fondamentali per la comprensione dell'esperienza delle audience che ne sono alla base. La liveness è un concetto ombrello 
sotto il quale si nasconde la relazione di liveness, ovvero quella relazione tra utenti, tecnologie ed eventi che modella l'esperienza che facciamo degli eventi dal vivo e che viviamo con diversi gradienti e diverse intensità. Parafrasando il titolo del testo di Van Es il futuro del live si sostanzia intorno ad un'esperienza della liveness che è mobile ed è figlia dei diversi formati generati dalla partecipazione ad un evento in tempo reale. Questo significa non solo che esistono formati di liveness digitali, quanto che tali formati si configurano a partire dalle diverse esperienze dell'evento che gli utenti fanno e che sono rette dalle diverse relazioni di liveness. Queste relazioni devono per forza di cose tenere conto delle possibilità di partecipazione e delle caratteristiche delle tecnologie sempre storicamente e culturalmente determinate e che ridefiniscono di volta in volta la relazione di liveness: un evento è dal vivo a seconda delle modalità di partecipazione che esso mi permette. Lungi dall'essere un concetto fuori dalle forme di mediazione, la relazione di liveness si basa su di esse: la relazione di liveness è una forma di mediazione anche quado tale mediazione è occulta e nascosta. Nel contesto del mediascape contemporaneo molte delle forme di liveness digitali possono essere pensate come forme di rimediazione di formati e relazioni di liveness precedentemente sorte in forme mediali analogiche precedenti (come ho provato a mostrare con l'esempio della tecnologia telefonica). Questo vuol dire che la radice dell'odierno dibattito sulla liveness va posizionata nelle tecnologie sorte alla fine dell'Ottocento e che, seguendo Mario Costa hanno prodotto un vero e proprio uomo fuori di sé ${ }^{37}$. Il tratto dell'impermanenza della liveness che abbiamo, in mancanza di altri termini, definito con il concetto di "Vanish liveness" (abbiamo deciso di usare la forma nominale piuttosto che la forma aggettivale perché pur suonando strana in inglese mostra come i processi di sparizione si innervino intorno ad oggetti specifici), nelle forme di liveness digitali può essere pensato come una rimediazione delle tradizionali relazioni di liveness di tipo teatrale. Questo non vuol dire che la liveness in quanto tale è figlia esclusivamente dell'impermanenza quanto che questo particolare modello di liveness è figlio della mancanza di forma archiviata dell'evento. La forma archiviata conserva i tratti della liveness dell'evento primario pur in forma simulata (ad esempio le dirette Facebook che viste in

\footnotetext{
${ }^{37}$ M. Costa, L'uomo fuori di sé. Alle origini della esternalizzazione: la fotografia, il fonografo e il telefono nella Parigi del XIX secolo, Mimesis, Milano 2018.
} 
forma archiviata conservano la scansione dei commenti nei momenti in cui essi sono stati postati dando l'impressione all'utente di vivere quell'esperienza dal vivo). La "vanish liveness" si differenzia dalle altre forme di liveness digitali perché di essa non esiste forma archiviata $^{38}$. Essa è mancante anche del resto prodotto dalla liveness simulata nell'archiviazione che trasforma l'evento in qualcosa di diverso: la sua relazione di liveness è monca perché in essa viene a mancare la condivisione di un tempo e non esiste più relazione temporale condivisa con l'utente e tecnologie.

L'esempio della tecnologia telefonica ci serve per mostrare come se da un lato la condivisione di uno spazio fisico, con tutto ciò che esso comporta in epoca digitale con la proliferazione di spazi digitali, non può essere un discrimine per determinare quale evento sia dal vivo rispetto a quale non lo sia, dall'altro lato rimane la questione della temporalità la cui condivisione rappresenta ancora un elemento fondamentale perché una determinata esperienza possa essere considerata dal vivo. In questi termini, è chiaro che ogni relazione di liveness porta con sé i germi dell'impermanenza, la possibilità di essere vanish, ma rispetto a questo, a mio parere, quelle forme in cui l'impermanenza diventa decisiva per l'esperienza degli utenti e che viene esibita come valore intrinseco dell'evento, sono profondamente differenti rispetto a quegli eventi in cui le forme di archiviazione simulano la relazione di liveness, questo perché si basano su diversi gradienti con cui facciamo esperienza degli eventi live.

\section{Riferimenti Bibliografici}

S. Arabatzis, Medienherrschaft, Medienresistenz und Medienanarchie. Archäologie der Medien und ihr neuer Gebrauch, Springer, Berlin 2017.

P. Auslander, Liveness. Performace in mediatized culture, Routledge, London \& New York, 1999.

P. Auslander, Live and Techologically Mediated performace, in T.C. Davis (ed.) The Cambrigde Companion to Performance Studies, Cambridge University press, Cambridge 2008, pp. 107-119.

\footnotetext{
${ }^{38}$ Questo termine viene qui usato seguendo la distinzione operata da Diana Taylor laddove per questa: il repertorio si differenzia dall'archivio. L'archivio è definito da tutte quelle forme di archiviazione della performance legate all'orizzonte mediale mentre il repertorio si definisce a partire da quelle forme di trasmissione del sapere che sono incarnate in un corpo come passi di danza e conoscenze attoriali e che si trasmettono attraverso una trasmissione del sapere body to body. D. Taylor, Performance, politica e memoria culturale, Artemide, Roma 2018.
} 
P. Auslander, Digital Liveness: A Historico-Philosophical Perspective, in «Journal of Performance and Art», vol. 34, n. 3, 2012, pp. 3-11.

S. Banford, G. Giannachi, Performing Mixed Reality, MIT press, Cambridge 2011.

C. Bertho-Lavenir, Innovation technique et société du spectacle : le théâtrophone à l'Exposition de 1889, in «Le Mouvement social», n. 149, pp. 59-59.

L. Bevione (a cura di), Interior sites project. II teatro di Cuocolo/Bosetti. IRAA Theatre, Titivillus, Pisa 2017.

H. Blau, The Human Nature of the Bot: A Response to Philip Auslander, in «PAJ: A Journal of Performance and Art», vol. 24, n. 1, January 2002, pp. 22-24.

G. Boccia Artieri, Mediatizzazione e network society: un programma di ricerca, in "Sociologia della comunicazione», n. 50, 2015, pp. 60-67.

G. Boccia Artieri, L. Gemini, Per un live dei corpi a distanza, in "Doppiozero», $1^{\circ}$ Maggio, 2020, https://www.doppiozero.com/materiali/un-live-dei-corpi-distanza (ultimo accesso $25 / 11 / 2021)$.

W. Boddy, Redefining the Home Screen: Technological Convergence as Trauma and Business Plan, in D. Thorburn, H. Jenkins (a cura di), Rethinking Media Change: The Aesthetics of Television, MIT press, Cambridge, 2003, pp. 191-200.

M. Costa, L'uomo fuori di sé. Alle origini della esternalizzazione: la fotografia, il fonografo e il telefono nella Parigi deI XIX secolo, Meltemi, Milano 2018.

N. Couldry, Liveness, "reality", and the mediated habitus from television to the mobile phone, in «Communication Review», vol. 7, n. 4, 2004, pp. 353-361.

N. Couldry, A. Hepp, A., The Mediated Construction of Reality, Polty Press, Cambridge 2017.

A. Cristell, Liveness and Recording in the Media, Routledge, London \& New York, 2012.

R. Cuocolo, R. Bosetti, Theatre on a line, http://www.teatrodidioniso.it/in-evidenza/theatreon-a-line-di-cuocolo-bosetti-per-il-teatro-della-tosse/ (ultimo accesso 30/11/2021).

A.I. De Benedictis, Radiodramma e arte radiofonica. Storia e funzioni della musica per radio in Italia, EDT, Torino 2005.

V. Del Gaudio, Liveness: presenza, immagine digitale e falsificazione, in A. Rabbito (a cura di), La cultura del falso. Inganni, illusioni e fake news, Meltemi, Milano 2020, pp. 437-447.

V. Del Gaudio, Interactive Tools Performance: Blast Theory between Media Theory, Performance Studies and Social Research, in «Comunicazioni Sociali», n. 1, 2021, pp. 79-86.

F. Deriu, Il Teatro È Un Medium? Questioni E Risposte In Prospettiva "Mediologica", in "Mantichora», n. 5, dicembre 2015, pp. 59-65. 
E. Fisher- Lichte, Estetica del performativo. Una teoria del teatro e dell'arte, Carocci, Roma, 2014.

L. Gemini, Liveness: le logiche mediali nella comunicazione dal vivo, "Sociologia della comunicazione», n. 51, 2016, pp. 43-63.

L. Gemini, Antidoto liveness. La performance dal vivo durante il Covid-19, in «Sociologia della comunicazione», n. 60, 2020, pp. 104-118.

L. Gemini, S. Brilli, F. Giuliani, Il dispositivo teatrale alla prova del Covid-19. Mediatizzazione, liveness e pubblici, in «Mediascapes Journal», n. 20, 2020, pp. 44-58.

G. Giannachi, N. Kaye, M. Shanks (a cura di), Archaeologies of Presence, Routledge, London 2013.

R. Grusin, Radical Mediation Cinema, estetica e tecnologie digitali, Pellegrini, Cosenza 2017.

B. Hadley, Theatre, social media and meaning making, Routledge, London \& New York 2017.

E. Hammelburg, \#stemfie: reconceptualising liveness in the era of social media, in «Tijdschrift Voor Mediageschiedenis», vol. 18, n. 1, 2015, pp. 85-100.

L. Jervis, Immersive Embodiment. Theatres of Mislocalized Sensation, Palgrave Macmillan, London \& New York 2019.

Kepler 452, Consegne // una performance da coprifuoco, https://kepler452.it/CONSEGNE (ultimo accesso 30/11/2021).

P. Levy, Il virtuale, Raffaello Cortina, Milano 1997.

P. Lonergan, Theatre \& Social Media, Palgrave Macmillan, London 2013.

A.M. Monteverdi, Leggere lo spettacolo multimediale, Dino Audino, Roma, 2020.

J. Parikka, Archeologia dei media. Nuove prospettive per la storia e la teoria della comunicazione, Carocci, Roma 2019.

P. Phelan, Unmarked. The politics of performance. Routledge, London \& New York 1994.

G. Pisano, The Théâtrophone, an Anachronistic Hybrid Experiment or One of the First Immobile Traveler Devices? In A. Gaudreault, N. Dulac, S. Hidalgo (a cura di), A Companion to Early Cinema, Blackwell Pub, Hoboken, 2012, pp. 80-98.

E. Pitozzi, (a cura di), «Culture Teatrali. On Presence», n. 21, 2011.

A. Pizzo, Teatro e mondo digitale. Attori, scena e pubblico, Venezia, Marsilio, 2003.

R. Sacchettini, La radiofonica arte invisibile. Il radiodramma italiano prima della televisione, Titivillus, Pisa 2011.

D. Sacco, Teatro come medium Sulla riproducibilità a partire da Walter Benjamin e Samuel Weber, in «Dradek», vol. III, n. 1-2, 2017, pp.87-109. 
P. Scandell, Television and the Meaning of Live: An Enquiry into the Human Situation, Polity, Malden 2014.

R. Schneider, It Seems as If...I Am Dead Zombie Capitalism and Theatrical Labor, in «TDR: The Drama Review», vol. 56, n. 4, Winter 2012, pp. 150-162.

P.W. Sewell, Television in the age of radio, Rutgers University press, London 2014.

D. Taylor, Performance, Politica E Memoria Culturale, Artemide, Roma 2018.

V. Valentini, Drammaturgie Sonore, Bulzoni, Roma 2012.

K. Van Es, The future of live, Polty Press, Cambridge, 2017.

K. Von Hagen, Devant le téléphone: Sinn und Sinnlichkeit oder das théâtrophone bei Proust, in U. Felden, V. Roloff (a cura di), Die Korrespondenz der Sinne. Wahrnehmungsästhetische und intermediale Aspekte im Werk von Proust, Brill, Berlin 2008, pp. 215-232.

M. White, Television and Internet Differences by Design: Rendering Liveness, Presence, and Lived Space. In «Convergence: The International Journal of Research into New Media Technologies», vol. 3, n. 12, 2006, pp. 341-55.

\section{Biografia dell'autore/ Author's biography}

Vincenzo Del Gaudio insegna Teorie e Tecniche dello spettacolo multimediale all'Università degli Studi di Salerno, Storia del teatro e dello spettacolo all'Università della Tuscia e Storia del teatro contemporaneo all'Università degli Studi eCampus. Si occupa dei rapporti tra teatro e sociologia e di sociologia del teatro e dei rapporti tra teatro e media digitali. Tre le sue ultime pubblicazioni figura Theatron. Verso una mediologia del teatro e della performance (Meltemi, Milano 2020). Ha scritto alcuni saggi su teatro e comunità in particolare sul territorio campano.

Vincenzo Del Gaudio teaches Theories and Techniques of Multimedia Performance at the University of Salerno, History of Theatre and Performing Arts at the University of Tuscia and History of Contemporary Theatre at eCampus University. His research interests deal with the relations between theater and sociology and with the relations between theater and digital media. Among his last publications there is Theatron. Towards a mediology of theater and performance (Meltemi, Milano 2020). He has written several essays on theater and community focused the territory of Campania.

Articolo sottoposto a double-blind peer review 


\title{
Livecasting without the live: The multiple temporalities of National Theatre at Home
}

\author{
Sarah Byrne \\ University of Reading
}

\begin{abstract}
Since the 2009 launch of NT Live, the National Theatre has been at the forefront of UK livecasting so the calls to respond to the complete lockdown of the UK in March 2020 weren't surprising. This paper will analyse how this pandemic response utilised the multiple temporalities of NT Live to create a theatrical event in viewers' homes. Despite using past recordings, the NT At Home programme was a response to a specific historical event and was structured around an "ontological now" that in return shaped the reception of those recordings. NT Live capitalised on the liveness and the exclusivity of their broadcasts. Performances were shown at a set time, streamed simultaneously into cinemas. NT At Home challenged every part of this system of liveness and in doing so created its own contradictory and fluid temporal identity. In this paper I will draw on liveness studies from multiple disciplines to cover three aspects of these new temporalities and present a case study of liveness practice.
\end{abstract}

Sin dal lancio nel 2009 di NT Live, il National Theatre è stato protagonista nel livecasting nel Regno Unito; non sorprende quindi l'aspettativa di una risposta dell'istituzione teatrale a seguito del lockdown di marzo 2020. Il presente articolo cerca di analizzare questa risposta pandemica da parte dell'NT Live abbia utilizzato molteplici temporalità per ricreare un senso dell'evento teatrale nelle case degli spettatori. Nonostante l'utilizzo di materiale d'archivio, l'iniziativa NT At Home ha costituito una risposta a uno specifico evento storico strutturata attorno un "ora ontologico" che modellato la ricezione di questo materiale: NT Live capitalizzava sulla liveness e sull'esclusività della trasmissione, con performance mostrate a un orario specifico e trasmesse simultaneamente al cinema; NT At Home rielabora invece questo sistema di liveness, creando il proprio sento di una temporalità fluida e contraddittoria. L'articolo attinge quindi ad approcci sulla liveness provenienti da molteplici discipline per esaminare tre aspetti in particolare di questa nuova temporalità e un caso di studio specifico di "pratica di liveness".

\section{Key Words}

National Theatre, Livecasting, Event Cinema, Streaming 